\title{
MicroRNA-103a curtails the stemness of non-small cell lung cancer cells by binding to OTUB1 through the Hippo signaling pathway
}

Zhenzhen Hu

Jiangxi Tumor Hospital

Dan Xiao

Jiangxi Tumor Hospital

Tingting Qiu

Jiangxi Tumor Hospital

Jun Li

Jiangxi Tumor Hospital

Zhentian Liu ( $\square$ Liuzhentian120902@163.com )

Jiangxi Tumor Hospital https://orcid.org/0000-0002-7436-8874

Research

Keywords: microRNA-103a; OTUB1, The Hippo pathway, Non-small cell lung cancer, Caner stem cells

Posted Date: May 17th, 2020

DOI: https://doi.org/10.21203/rs.3.rs-27754/v1

License: (c) (i) This work is licensed under a Creative Commons Attribution 4.0 International License. Read Full License 


\section{Abstract}

Background Although dysfunction of microRNA-103a (miR-103a) has been implicated in various cancers, its relevance in non-small cell lung cancer (NSCLC) is unsettled. This study was designed with an aim to examine the molecular mechanism underlying the regulatory role of miR-103a in NSCLC.

Methods Kaplan-Meier analysis was carried out to study the correlation between overall survival of NSCLC patients and miR-103a expression. RT-qPCR and Western blot were applied to evaluate the expression of relevant genes in tissues and cells. Sphere formation, MTS, flow cytometry as well as Transwell assays were conducted for characterizing the stemness. Dual-luciferase reporter gene assays were applied to clarify the binding relationship between miR-103a and ovarian tumor domain-containing ubiquitin aldehyde binding protein 1 (OTUB1).

Results miR-103a expression was diminished in NSCLC tissues and cells, whereas OTUB1 expression was increased. Higher miR-103 expression indicated better prognosis for patients with NSCLC. After overexpression of miR-103a, the cell viability and stemness were diminished, while the cycle arrest and apoptosis rate were facilitated, and the expression of p-YAP decreased significantly. The opposite trends were observed after miR-103a silencing. miR-103a lowered the expression of OTUB1, while overexpression of OTUB1 blocked the inhibition effects of miR-103a on NSCLC.

Conclusion miR-103a/OTUB1/Hippo axis plays a possible role in modulating the malignant behavior and stemness of cells which might function as a possible therapeutic option for the management of NSCLC.

\section{Introduction}

Lung cancer, a kind of heterogeneous tumor, could be divided into non-small cell lung carcinoma (NSCLC, contributing to $80-85 \%$ of all lung cancer cases) and small cell lung carcinoma [1]. In the United States, lung cancer is estimated to be responsible for almost one-quarter of all cancer-related deaths (about 135,720 deaths) in 2020 [2]. In general, lung cancer patients at early stage are treated with surgery, while patients at advanced stage or with metastases undergo chemotherapy [3]. The 5-year survival rate for patients with lung cancer in the United States is $15.6 \%$, and even though some enhancements in survival have been achieved during the past few decades, the advances was not as substantial as that in other malignancies [4]. Cancer stem cells (CSCs) are defined as the reservoir of tumor cells since they display the property of self-renewal and the competence of reestablishing a heterogeneous cancer cell population [5]. Therefore, clarification of the molecular mechanisms of CSCs might offer novel insights for the treatments of NSCLC.

MicroRNAs (miRNAs) are noncoding RNAs of about $22 \mathrm{nt}$ in length, and dysfunction of miRNAs may participate in various events of tumorigenesis by mediating cell cycle, apoptosis and migration [6]. Interestingly, several miRNAs, including miR-124a and miR-181b have been reported to inhibit stemness of CSCs and to overcome drug resistance in NSCLC [7, 8]. In addition, miR-103a-3p expression was remarkably reduced in NSCLC tissues and cell lines, which was tightly correlated with dismal survival of 
NSCLC sufferers [9]. Moreover, miR-103a-3p was engaged in the tumor-suppressor role of linc00152 depletion in glioma stem cells [10]. However, its role in CSCs of lung cancer has yet to be clarified. As a consequence, we postulated that miR-103a exerts regulatory functions over CSCs in NSCLC. Located within locus 11q13.1, ovarian tumor domain-containing ubiquitin aldehyde-binding protein 1 (OTUB1) is expressed in a great range of human tissues, and OTUB1 knockdown in the A549 cells suppressed soft agar colony formation and xenograft tumor growth [11]. Besides, OTUB1 was revealed as a biomarker in the pathogenesis of gliomas and has the potency to be applied as a clinical biomarker for glioma in the future [12]. In this report, the expression pattern of miR-103a in NSCLC patients was surveyed to determine the prognostic values of miR-103a in NSCLC. Moreover, we revealed the probable molecular mechanism of miR-103a in CSCs of NSCLC through regulating OTUB1.

\section{Material And Methods}

\section{Collection of tissue samples}

NSCLC tissues and adjacent normal lung tissues (at least $5 \mathrm{~cm}$ away from tumor tissues) were harvested from 73 patients diagnosed with primary NSCLC and underwent tumor resection at Jiangxi Tumor Hospital from December 2013 to June 2015. None patients received radiotherapy or chemotherapy before surgery. Patients combined with other chronic diseases were excluded. The obtained tissues were snap frozen and kept in liquid nitrogen at $-80^{\circ} \mathrm{C}$ until later experiments. The patients were visited every month to collect and record the prognostic survival. The detailed information of all patients is listed in Table 1.

Table 1

Detailed characteristics of the 73 patients

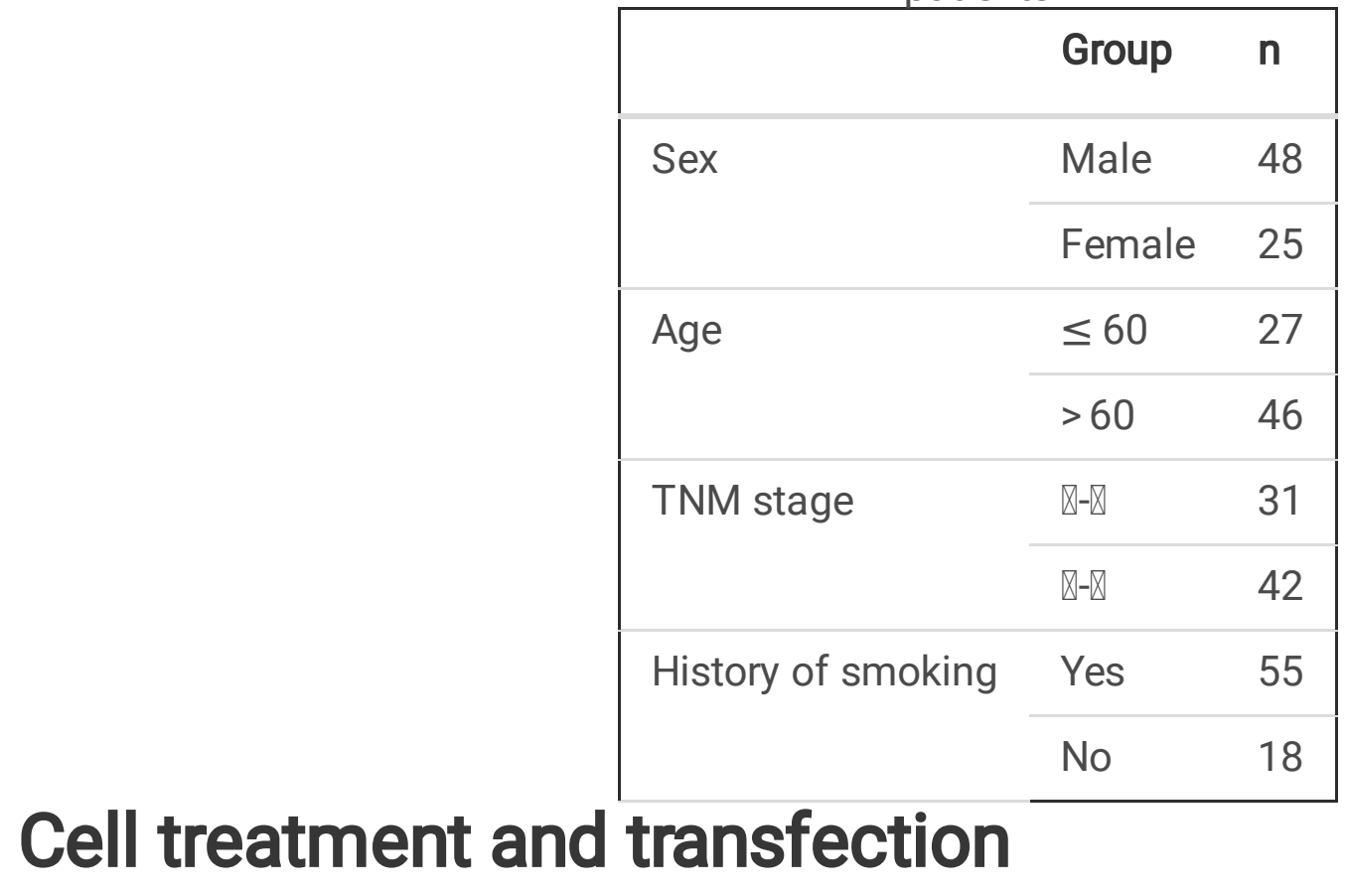


The cultured two normal lung epithelial cell lines Beas-2B and HBE, NSCLC cell lines 95D, A549, NCl-H520, $\mathrm{NCl}-\mathrm{H} 460$ and H1299 as well as human embryonic kidney 293T (HEK293T) cells were from ATCC. No mycoplasma contamination was confirmed by the cellosaurus query website. Normal lung epithelial cell lines Beas-2B and HBE were cultured in LHC-9 medium. 95D, A549, NCI-H520, NCI-H460, H1299 and HEK293T cells were grown in Dulbecco's modified Eagle's medium (DMEM, Invitrogen, Carlsbad, CA) with $1 \%$ penicillin/streptomycin (Invitrogen) and 10\% fetal bovine serum (HyClone, Logan, UT) in a $5 \% \mathrm{CO}_{2}$ incubator at $37^{\circ} \mathrm{C}$.

The A549 cells were trypsinized and resuspended into $1 \times 10^{3}$ cells $/ \mathrm{mL}$ single cell suspension with serumfree DMEM/F12 culture medium containing epidermal growth factor, basic fibroblast growth factor, insulin, bovine serum albumin, B27 and glucose. The culture flask was vertically placed in the incubator and shaken 5 times a day to observe the formation of microspheres. Half of the medium was renewed once every 2 days, and the cells were passaged every 6 days. The microspheres in logarithmic growth phase were collected, detached with Accutase, triturated into single cells and incubated with Hoechst 33342. Flow cytometry was applied to detect the side population (SP) cells. When the proportion of the SP cells reached $25 \%$, all spheres were collected and made into a single cell suspension with phosphate buffered saline (PBS). The cell suspension was incubated with the monoclonal antibody labeled with fluorescence to sort $\mathrm{CD} 133^{+}$and $\mathrm{CD} 44^{+}$cells as A549 stem cells with a fluorescent activated cell sorter.

Negative control (NC) mimic, miR-103a mimic, NC inhibitor, miR-103a inhibitor, overexpressed (oe)-NC, oeOTUB1 vectors were generated by Life technologies (Grand Island, NY) and delivered into the cells to a final concentration at $20 \mathrm{nM}$. According to the instructions for FuGENE6 transfection reagent (Promega, Madison, WI), A549 cells were cultured overnight and transfected once reaching $60 \%$ confluence. Cells were collected $24 \mathrm{~h}$ or $48 \mathrm{~h}$ after transfection.

\section{Reverse transcription quantitative polymerase chain reaction (RT-qPCR)}

Total RNA was extracted from cells and tissues using Trizol (Invitrogen). Nanodrop spectrophotometer 2000 (1011U, NanoDrop Technologies, Wilmington, DE) was applied to determine the concentration and purity of total RNA. The reverse transcription was performed according to the TaqMan MicroRNA Assays Reverse Transcription primer (4427975, Applied Biosystems, Inc., Foster City, CA) instructions to produce cDNA. The qPCR primers were all commissioned to Sangon Biological Engineering Technology \& Services Co., Ltd. (Shanghai, China) for synthesis (Table 2). ABI 7500 quantitative PCR instrument (7500, ABI, Oyster Bay, NY) was used for real-time fluorescence quantitative PCR detection. U6 and glyceraldehyde-3phosphate dehydrogenase (GAPDH) were used as endogenous controls for detection of miRNA and gene expression, respectively. Relative quantification $\left(2^{-\Delta \Delta C t}\right)$ method was used for fold changes' calculating. 
Table 2

Primer sequence

\begin{tabular}{|ll}
\hline Targets & Sequence (5' $\left.{ }^{\prime} \mathbf{3}^{\prime}\right)$ \\
\hline miR-103a & F: AGCAGCATTGTACAGGGCTAT \\
& R: GTGCAGGGTCCGAGGT \\
OTUB1 & F: TCCTACAGGTCTCAGGTCCG \\
& R: GCTCTGACACCAGAGGGTTC \\
U6 & F: CTCGCTTCGGCAGCACA \\
& R: AACGCTTCACGAATTTGCGT \\
GAPDH & F: GAAGGTGAAGGTCGGAGT \\
& R: GAAGATGGTGATGGGATTTC
\end{tabular}

Note: miR-103a, microRNA-103a; OTUB1, ovarian tumor domain-containing ubiquitin aldehyde binding protein 1; GAPDH, glyceraldehyde-3-phosphate dehydrogenase.

\section{3-(4,5-dimethylthiazol-2-yl)-5-(3-carboxymethoxyphenyl)-2- (4-sulfophenyl)-2H-tetr-azolium (MTS) assay}

A549 cells were plated in a 96-well plate (Corning Co, Corning, NY) at a rate of $1 \times 10^{3}$ cells each well and cultured with $5 \% \mathrm{CO}_{2}$ at $37^{\circ} \mathrm{C}$. The cell viability was detected in each group using a CellTiter96 Aqueous One Solution Cell Proliferation Assay Kit (Promega) after $24 \mathrm{~h}$ of cell transfection. The cells were grown for $2 \mathrm{~h}$ with MTS reagent at $37^{\circ} \mathrm{C}$ with $5 \% \mathrm{CO}_{2}$. The optical density (OD) value at $490 \mathrm{~nm}$ was measured using a SpectraMax 340PC384 microplate reader to assess cell proliferation.

\section{Flow cytometry}

After $48 \mathrm{~h}$ of transfection, the cells at the confluence of $80 \%-90 \%$ were detached with trypsin and fixed with $70 \%$ ethanol in PBS at $-20^{\circ} \mathrm{C}$ overnight. Cell suspension $\left(1 \times 10^{6} \mathrm{cells} / \mathrm{mL}\right)$ was incubated with $50 \mu \mathrm{g} / \mathrm{mL}$ propidium iodide in PBS avoiding light for $10 \mathrm{~min}$. The apoptosis and cycle distribution of the cells was determined using an Attune NxT flow cytometer (Thermo Fisher Scientific Inc., Waltham, MA).

\section{Determination of cell migration and invasion}

Matrigel (Corning) was diluted with Roswell Park Memorial Institute (RPMI)-1640 medium (Solarbio, Beijing, China) and added into the chamber dropwise. Cells suspended in RPMI-1640 medium $\left(1 \times 10^{4}\right.$ cells $/ \mathrm{mL}$ ) were seeded into 6-well plates, while $500 \mu \mathrm{L}$ RPMI-1640 medium supplemented with $10 \%$ fetal bovine serum was placed in the basolateral chamber. After an incubation for $24 \mathrm{~h}$ at $37^{\circ} \mathrm{C}$ with $5 \% \mathrm{CO}_{2}$, the cells in the basolateral chamber were washed with PBS, fixed in $4 \%$ paraformaldehyde for $30 \mathrm{~min}$ at room temperature and stained with $0.5 \%$ crystal violet staining solution for $30 \mathrm{~min}$. After being fixed with neutral gum (Sigma-Aldrich Chemical Company, St Louis, MO, USA), five visual fields were randomly 
selected under an inverted microscope (Eclipse Ti, Nikon, Japan) for photograph and calculating the invaded cell number. Matrigel was not added in the cell migration assay, and the other steps were the same as the invasion assay.

\section{Sphere formation assay}

The pretreated cells were resuspended in DMEM/F12 medium (500-1000 cells/mL) and then cultured for 12 days in 6-well ultra-low attachment petri dishes (Corning). The number and size of tumor spheres were counted and measured using a contrast microscope (EVOS M7000, Nikon, Japan) at the 12th day to analyze the growth of tumor spheres.

\section{Western blot}

After $48 \mathrm{~h}$ of cell transfection, the cells were lysed on ice with radio immunoprecipitation assay solution, and centrifuged at $20000 \times \mathrm{g}$ for $10 \mathrm{~min}$ at $4{ }^{\circ} \mathrm{C}$ to gather the supernatant. Subsequently, $15 \mu \mathrm{g}$ total protein was subjected to $12 \%$ sodium dodecyl sulfate polyacrylamide gel electrophoresis, and then electroblotted onto a nitrocellulose membrane. Protein concentrations were determined with a bicinchoninic acid assay kit (Abcam Inc., Cambridge, UK). The proteins were then incubated with primary rabbit antibodies against OTUB1 (ab175200), YAP (ab52771) and p-YAP (ab76252) as well as goat antirabbit IgG (ab97051) at $4{ }^{\circ} \mathrm{C}$. All antibodies were from Abcam. Immunoreactive bands were measured using the enhanced chemiluminescence detection Kit (Thermo Fisher Scientific).

\section{Dualluciferase reporter assay}

We synthesized oligonucleotides in OTUB1 mRNA 3'untranslated region (3'UTR) containing targeting sequences with miR-103a. Meanwhile, miR-103a overexpression vector, inhibitor vector and vector containing miR-103a mutation were designed. The targeting sites were mutated to construct miR-103a mutant. The PGLO vector was selected to construct the fluorescence reporter vector pGLO-OTUB1. The vectors were extracted using a plasmid purification kit (Invitrogen). 293T cells were cultivated in a 24-well plate. The $200 \mathrm{ng}$ pGLO-OTUB1 plasmid and $20 \mathrm{nM}$ miR-103a mimic, inhibitor or mutant were cotransfected for a total of $24 \mathrm{~h}$ before the cell lysates were collected and the luciferase activity was determined by the dual luciferase reporter system (E1910, Promega). Firefly luciferase activity was used as a normalizer.

\section{Statistics}

SPSS 22.0 statistical software (IBM Corp. Armonk, N.Y., USA) was applied for all statistical analysis. All data are displayed as the mean \pm standard deviation. Comparisons between two groups were analyzed by paired $t$ test (between normal tissues and tumor tissues) or unpaired $t$ test (other two groups), while comparisons among multiple groups were assessed by one-way or two-way analysis of variation (ANOVA), followed by Tukey's post hoc test. The survival of patients was evaluated using Kaplan-Meier analysis. $p$ value $<0.05$ was symbolic of a significant difference.

\section{Results}




\section{MiR-103a is reduced in NSCLC tissues and cells}

We collected 73 samples of NSCLC tissues and matched normal lung tissues and adopted RT-qPCR to assess the miR-103a expression in tissues samples. As shown in Fig. 1A, miR-103a was decreased in tumor tissues versus normal tissues. Then, we divided patients into high miR-103a expression and low miR-103a expression groups according to the median value of miR-103 in 73 patients. Subsequently, the survival analysis in NSCLC patients was conducted, which manifested that miR-103a expression was closely correlated to the overall survival rate, and the survival rate of patients harboring high expression of miR-103a was higher than that of patients with low expression of miR-103a (Fig. 1B). Next, we examined the expression of miR-103a in Beas-2B, HBE, 95D, A549, NCl-H520, NCl-H460, H1299 cell lines. Compared with Beas-2B and HBE cells, the expression of miR-103a was significantly reduced in NSCLC cell lines (Fig. 1C). Therefore, the expression of miR-103a may link to the occurrence of NSCLC. To further explore the regulatory relationship between miR-103a and CSCs in NSCLC, we selected A549 cells to screen and identify SP cells for subsequent experiments (Fig. 1D-F).

\section{miR-103a inhibits stemness of CSCs in NSCLC}

To confirm whether the expression of miR-103a affects the biological properties of CSCs in NSCLC. We overexpressed and silenced miR-103a in sorted CSCs. We tested the miR-103a expression in each group of cells by RT-qPCR, which displayed that miR-103a expression was remarkably increased or decreased by miR-103a mimic or inhibitor versus their respective controls (Fig. 2A). We then assessed the activities of proliferation, cell cycle distribution, apoptosis, migration, invasion and sphere formation of the cells by MTS, flow cytometry, scratch test, Matrigel invasion assay and sphere formation assays, respectively. As shown in Fig. 2B, cell proliferation was repressed by miR-103a mimic, while promoted by miR-103a inhibitor. In addition, as shown in Fig. 2C-D, relative to the NC mimic treatment, the cell cycle of miR-103a mimic-treated cells was blocked in the G0/G1 phase, and the apoptosis was markedly increased. In comparison with the NC inhibitor delivery, the proportion of cells delivered with miR-103a inhibitor in the G0/G1 phase was significantly decreased, and the cell apoptosis was reduced. Moreover, ectopic expression of miR-103a diminished the cell migration and invasion, whereas miR-103a depletion contributed to opposite trends (Fig. 2E-F). Furthermore, overexpression of miR-103a decreased the sphere size and number of the cells, yet miR-103a inhibitor facilitated the formation of spheres in both number and size (Fig. 2G).

The impact of the Hippo signaling pathway on the development of NSCLC has been widely accepted. To explore whether miR-103a could affect the Hippo signaling pathway, we measured the expression of YAP and the extent of YAP phosphorylation in cells via western blot assays (Fig. $2 \mathrm{H}$ ). The expression of YAP showed insignificant difference in cells of each group. However, the extent of YAP phosphorylation was significantly attenuated by miR-103a mimic, while enhanced by miR-103a inhibitor versus their relative controls. These results illustrated that miR-103a reduces the biological properties of CSCs with the engagement of the Hippo signaling in NSCLC. 


\section{OTUB1 is directly targeted and inhibited by miR-103a in NSCLC cells}

Subsequently, we examined OTUB1 mRNA expression in NSCLC and adjacent normal lung tissue samples by RT-qPCR, and found that OTUB1 expression was increased in NSCLC tissue samples (Fig. 3A). We then performed a correlation analysis of the expression of miR-103a and OTUB1 mRNA in NSCLC tissue samples, and also observed a significant negative correlation (Fig. 3B). As predicted by the bioinformatic website, there exists a complementary sequence between miR-103a and OTUB1 (Fig. 3C). To further confirm the effect of miR-103a on translation of OTUB1 mRNA, a dual-luciferase reporter assay was performed. Most importantly, compared with NC treatment, miR-103a mimic reduced the luciferase activity of PGLO-OTUB1 reporter vector, while miR-103a inhibitor increased its luciferase activity, whereas mutant vector exerted no significant impact on the luciferase activity of PGLO-OTUB1 reporter vector (Fig. 3D). Therefore, we also tested the OTUB1 mRNA and protein expression in cells after transfection by RT-qPCR (Fig. 3E) as well as western blot assays (Fig. 3F-G). The expression of OTUB1 was diminished by miR-103a mimic compared to NC mimic, and significantly increased by miR-103a inhibitor compared with NC inhibitor.

\section{OTUB1 reverses miR-103a-induced repression on stemness of CSCs in NSCLC}

To further confirm whether the regulation of OTUB1 by miR-103a affects the development of CSCs in NSCLC, we transfected A549 cells with NC mimic + oe-NC, miR-103a mimic + oe-NC, NC mimic + oeOTUB1 or miR-103a mimic + oe-OTUB1, respectively. We first measured the expression of miR-103a and OTUB1 in cells after co-transfection by RT-qPCR and western blot (Fig. 4A-B). Compared with cells treated with NC mimic + oe-NC, the cells treated with miR-103a mimic + oe-NC displayed elevated miR-103a expression and downregulated OTUB1 mRNA and protein expression, while the cells treated with NC mimic + oe-OTUB1 exhibited boosted OTUB1 mRNA and protein expression. Relative to the miR-103a mimic + oe-NC treatment, the OTUB1 expression was significantly increased after miR-103a mimic + oeOTUB1 treatment. As expected, in comparison to the NC mimic + oe-NC delivery, proliferation was remarkably decreased (Fig. 4C), cell cycle arrest (Fig. 4D) and apoptosis (Fig. 4E) were accelerated, while invasion (Fig. 4F), migration (Fig. 4G) and the number and the size of spheres (Fig. 4H) were downregulated by miR-103a mimic + oe-NC introduction. On the contrary, NC mimic + oe-OTUB1 showed opposite effects. More interestingly, the regulatory effects of miR-103a overexpression were strongly abrogated by the co-transfection of OTUB1 overexpression plasmids.

Lastly, we investigated whether miR-103a modulated the Hippo signaling pathway through OTUB1. Western blot (Fig. 4I) results revealed that compared with the NC mimic + oe-NC, the extent of YAP phosphorylation was diminished by miR-103a mimic + oe-NC group, and significantly increased by NC mimic + oe-OTUB1. Moreover, the level of phosphorylated YAP was upregulated in miR-103a and OTUB1 
overexpressing cells. Thus, it was demonstrated that miR-103a acted as a tumor suppressor that inhibited stemness of CSCs in NSCLC by activating the Hippo signaling pathway through OTUB1.

\section{Discussion}

The overall survival rate for lung cancer is projected to be one of the lowest (19\%) just behind pancreas cancer ( $9 \%$ ) and liver cancer (18\%) in 2020 for all stages combined [2]. CSCs, characterized by the capacities of self-renewal and differentiation, function significantly in the development of different kinds of cancers, such as melanomas and glioma [13-15]. Thus, thorough comprehension of the mechanisms of the maintenance of CSC stemness is of great importance for the development of anti-cancer therapeutic strategies [16]. Besides, the approach developed by Herreros-Pomares et al. allowed the application of lung CSCs, which provides novel insights into the field of CSCs in NSCLC [17]. By now, no powerful treatment targeting CSCs has been found, it is therefore even more vital to explore various CSC targeting measures [14]. Here, we illustrated that miR-103a expression was markedly reduced in NSCLC tissues and cell. In addition, the downregulation of miR-103a was correlated to the unsatisfactory survival of NSCLC patients. Furthermore, restoration of miR-103a suppressed CSC viability, migration, invasion in addition to sphere formation, whereas potentiated apoptosis in NSCLC. OTUB1 was recognized as a target of miR-103a. Our data further indicated that miR-103a repressed CSC cell self-renewal and stemness through the Hippo signaling by targeting OTUB1 in NSCLC.

In the current project, we firstly validated a significant downregulation of miR-103a in NSCLC clinical samples and cell lines. A previous work found that miR-103a was diminished in both gastric cancer cells and clinical cancer specimens and was linked to tumor, node, metastases stage of gastric cancer [18]. Besides, miR-103a-3p was poorly expressed in bladder cancer and promoted cell proliferation and migration via the interaction with CDK6 [19]. Moreover, miR-103a mimic led to reduced CSC viability, migration, invasion as well as sphere formation in this report, whereas the transfection of miR-103a inhibitor resulted in opposite trends. In a similar manner, miR-103a-3p repressed growth and metastasis through regulating the KRAS signaling and epithelial-to-mesenchymal transition in NSCLC cells [20]. While in the current work, we clarified the miR-103a mimic lowered the extent of YAP phosphorylation in NSCLC cells, indicating a correlation between miR-103a and the Hippo signaling pathway. YAP, a related mammalian homolog of Drosophila yorkie that acts as an effector of the Hippo signaling pathway, is often induced in malignancies and may exerted tumor-promoting properties by activating CSCs [21]. Moreover, YAP regulates key cellular functions, including proliferation control, suppression of apoptosis, and enhancement of metastasis in many tissues, and the Hippo signaling works as an important tumorsuppressor by negatively regulating oncogenic YAP [22]. Based on the above, poorly expressed miR-103a was associated with the maintenance of CSC stemness through the dysfunction of the Hippo signaling pathway.

Then, we applied an online software to identify the molecular targets of miR-103a. Interestingly, OTUB1 harbored putative binding sites with miR-103a. Besides, OTUB1 was monitored to be overexpressed in NSCLC cells and tissues. Consistent with our study, expression of OTUB1 was profoundly higher in colon 
cancer tissues than that in the matched normal tissues, which was correlated with tumor size and lymph node metastasis of patients with colon cancer [23]. Wound-healing and Transwell assays conducted by Weng et al. revealed that OTUB1 exerted pro-migration and pro-invasive capacities in gastric cancer [24]. In addition, OTUB1 was identified as an oncogene accelerating the progression and correlated with pathogenesis of hepatocellular carcinoma [25]. In the same vein, NC mimic + oe-OTUB1 treatment in our study contributed to facilitated cell viability, migration, invasion, cell cycle entry and sphere formation, whilst suppressed cell apoptosis. Also, upregulation of OTUB1 flattened the inhibition on cell proliferation, migration and invasion along with enhancement of apoptosis induced by miR-524-3p, implying that miR524-3p blocked colorectal cancer cell mobility by directly downregulating OTUB1 [26]. Esophageal cancer cell migration and invasion repressed by miR-542-3p was also at least partly attenuated by introduction of recombinant OTUB1 [27]. Our rescue experiments provided forceful evidence supporting that OTUB1 abrogated the carcinostatic role of miR-103a on CSC viability, migration, invasion and sphere formation in NSCLC. Moreover, we observed that miR-103a held the capacity to activate the Hippo signaling pathway by impairing the YAP phosphorylation in an OTUB1-depedent manner. Nevertheless, the detailed mechanisms through which OTUB1 governs the Hippo signaling need to be discovered in the future work. Also, this work just accomplishes in vitro assays, additional in vivo experiments are warranted to confirm our supposition.

\section{Conclusion}

To put it briefly, our study provides evidence that high expression of miR-103a reduces the proliferation, sphere formation, invasion and migration of CSC cells and promotes the apoptosis in NSCLC via downregulation of OTUB1 and the activation of the Hippo signaling pathway (Figure 5). This paper proposes that this axis might by amenable to therapeutic intervention of NSCLC.

\section{Abbreviations}

ANOVA, analysis of variation; CSCs, cancer stem cells; DMEM, Dulbecco's modified Eagle's medium; GAPDH, glyceraldehyde-3-phosphate dehydrogenase; HEK293T, human embryonic kidney 293T; MTS, 3(4,5-dimethylthiazol-2-yl)-5-(3-carboxymethoxyphenyl)-2-(4-sulfophenyl)-2H-tetr-azolium; NC, negative control; miR-103a, microRNA-103a; NSCLC, non-small cell lung cancer; OD, optical density; oe, overexpression; oe, overexpressed; OTUB1, ovarian tumor domain-containing ubiquitin aldehyde binding protein 1; PBS, phosphate buffered saline; RPMI, Roswell Park Memorial Institute; RT-qPCR, reverse transcription quantitative polymerase chain reaction; SP, side population; 3'UTR, 3'untranslated region.

\section{Declarations}

\section{Acknowledgements}

Not applicable. 


\section{Authors' contributions}

Zhenzhen Hu conceived the study and participated in its design and coordination; Dan Xiao and Tingting Qiu performed all experiments; Jun Li and Zhentian Liu analyzed and interpreted the data; The draft was improved through discussion and editing by all the authors who read and approved the final manuscript.

\section{Funding}

Not applicable.

\section{Availability of data and materials}

All the data generated or analyzed during this study are included in this published article.

\section{Ethics approval and consent to participate}

All patients approved to be enrolled by providing written informed consent. The study was accepted by the Ethical Committee of Jiangxi Tumor Hospital, and complied with the Declaration of Helsinki.

\section{Consent for publication}

Not applicable.

\section{Competing interests}

The authors declare that they have no competing interests.

\section{References}

1. Osmani L, Askin F, Gabrielson E, Li QK. Current WHO guidelines and the critical role of immunohistochemical markers in the subclassification of non-small cell lung carcinoma (NSCLC): Moving from targeted therapy to immunotherapy. Semin Cancer Biol. 2018;52(Pt 1):103-109.

2. Siegel RL, Miller KD, Jemal A. Cancer statistics, 2020. CA Cancer J Clin. 2020;70(1):7-30.

3. Lu T, Yang X, Huang Y, Zhao M, Li M, Ma K, et al. Trends in the incidence, treatment, and survival of patients with lung cancer in the last four decades. Cancer Manag Res. 2019;11943-953.

4. Dela Cruz CS, Tanoue LT, Matthay RA. Lung cancer: epidemiology, etiology, and prevention. Clin Chest Med. 2011;32(4):605-644.

5. Bighetti-Trevisan RL, Sousa LO, Castilho RM, Almeida LO. Cancer Stem Cells: Powerful Targets to Improve Current Anticancer Therapeutics. Stem Cells Int. 2019;20199618065.

6. Liao J, Shen J, Leng Q, Qin M, Zhan M, Jiang F. MicroRNA-based biomarkers for diagnosis of nonsmall cell lung cancer (NSCLC). Thorac Cancer. 2020. 
7. Wang X, Meng Q, Qiao W, Ma R, Ju W, Hu J, et al. miR-181b/Notch2 overcome chemoresistance by regulating cancer stem cell-like properties in NSCLC. Stem Cell Res Ther. 2018;9(1):327.

8. Yu F, Liu JB, Wu ZJ, Xie WT, Zhong XJ, Hou LK, et al. Tumor suppressive microRNA-124a inhibits stemness and enhances gefitinib sensitivity of non-small cell lung cancer cells by targeting ubiquitinspecific protease 14. Cancer Lett. 2018;42774-84.

9. She Y, Han Y, Zhou G, Jia F, Yang T, Shen Z. hsa_circ_0062389 promotes the progression of nonsmall cell lung cancer by sponging miR-103a-3p to mediate CCNE1 expression. Cancer Genet. 2020;24112-19.

10. Yu M, Xue Y, Zheng J, Liu X, Yu H, Liu L, et al. Linc00152 promotes malignant progression of glioma stem cells by regulating miR-103a-3p/FEZF1/CDC25A pathway. Mol Cancer. 2017;16(1):110.

11. Saldana M, VanderVorst K, Berg AL, Lee H, Carraway KL. Otubain 1: a non-canonical deubiquitinase with an emerging role in cancer. Endocr Relat Cancer. 2019;26(1):R1-R14.

12. Xu L, Li J, Bao Z, Xu P, Chang H, Wu J, et al. Silencing of OTUB1 inhibits migration of human glioma cells in vitro. Neuropathology. 2017;37(3):217-226.

13. Papaccio F, Paino F, Regad T, Papaccio G, Desiderio V, Tirino V. Concise Review: Cancer Cells, Cancer Stem Cells, and Mesenchymal Stem Cells: Influence in Cancer Development. Stem Cells Transl Med. 2017;6(12):2115-2125.

14. Akbari-Birgani S, Paranjothy T, Zuse A, Janikowski T, Cieslar-Pobuda A, Likus W, et al. Cancer stem cells, cancer-initiating cells and methods for their detection. Drug Discov Today. 2016;21(5):836-842.

15. Vlashi E, Pajonk F. Cancer stem cells, cancer cell plasticity and radiation therapy. Semin Cancer Biol. 2015;3128-35.

16. Gu J, Zhang Z, Lang T, Ma X, Yang L, Xu J, et al. PTPRU, As A Tumor Suppressor, Inhibits Cancer Stemness By Attenuating Hippo/YAP Signaling Pathway. Onco Targets Ther. 2019;128095-8104.

17. Herreros-Pomares A, de-Maya-Girones JD, Calabuig-Farinas S, Lucas R, Martinez A, Pardo-Sanchez $\mathrm{JM}$, et al. Lung tumorspheres reveal cancer stem cell-like properties and a score with prognostic impact in resected non-small-cell lung cancer. Cell Death Dis. 2019;10(9):660.

18. Liang J, Liu X, Xue H, Qiu B, Wei B, Sun K. MicroRNA-103a inhibits gastric cancer cell proliferation, migration and invasion by targeting c-Myb. Cell Prolif. 2015;48(1):78-85.

19. Zhong Z, Lv M, Chen J. Screening differential circular RNA expression profiles reveals the regulatory role of circTCF25-miR-103a-3p/miR-107-CDK6 pathway in bladder carcinoma. Sci Rep. 2016;630919.

20. Fan Z, Yang J, Zhang D, Zhang X, Ma X, Kang L, et al. The risk variant rs884225 within EGFR impairs miR-103a-3p's anti-tumourigenic function in non-small cell lung cancer. Oncogene. 2019;38(13):2291-2304.

21. Snigdha K, Gangwani KS, Lapalikar GV, Singh A, Kango-Singh M. Hippo Signaling in Cancer: Lessons From Drosophila Models. Front Cell Dev Biol. 2019;785.

22. Ehmer U, Sage J. Control of Proliferation and Cancer Growth by the Hippo Signaling Pathway. Mol Cancer Res. 2016;14(2):127-140. 
23. Liu X, Jiang WN, Wang JG, Chen H. Colon cancer bears overexpression of OTUB1. Pathol Res Pract. 2014;210(11):770-773.

24. Weng W, Zhang Q, Xu M, Wu Y, Zhang M, Shen C, et al. OTUB1 promotes tumor invasion and predicts a poor prognosis in gastric adenocarcinoma. Am J Transl Res. 2016;8(5):2234-2244.

25. Ni Q, Chen J, Li X, Xu X, Zhang N, Zhou A, et al. Expression of OTUB1 in hepatocellular carcinoma and its effects on HCC cell migration and invasion. Acta Biochim Biophys Sin (Shanghai). 2017;49(8):680-688.

26. Yuan L, Yuan P, Yuan H, Wang Z, Run Z, Chen G, et al. miR-542-3p inhibits colorectal cancer cell proliferation, migration and invasion by targeting OTUB1. Am J Cancer Res. 2017;7(1):159-172.

27. Sun J, Deng Y, Shi J, Yang W. MicroRNA5423p represses OTUB1 expression to inhibit migration and invasion of esophageal cancer cells. Mol Med Rep. 2020;21(1):35-42.

\section{Figures}

A

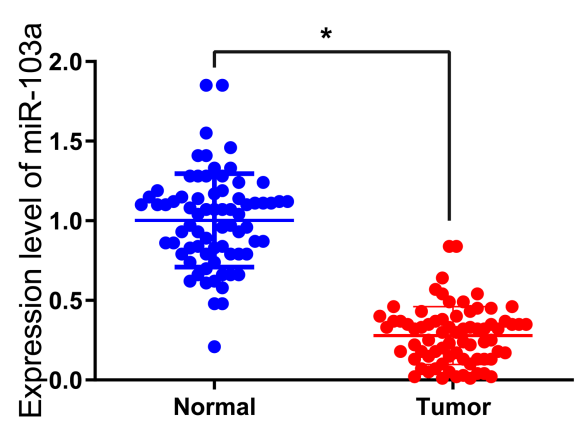

D

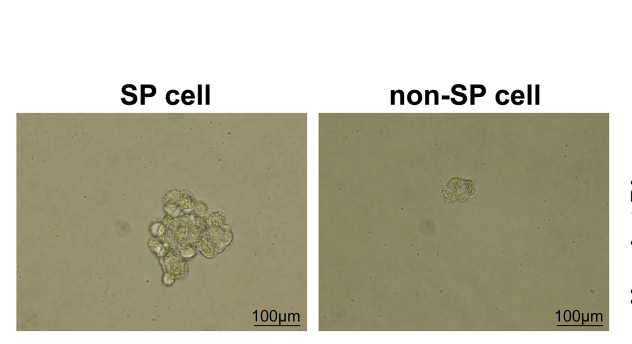

B

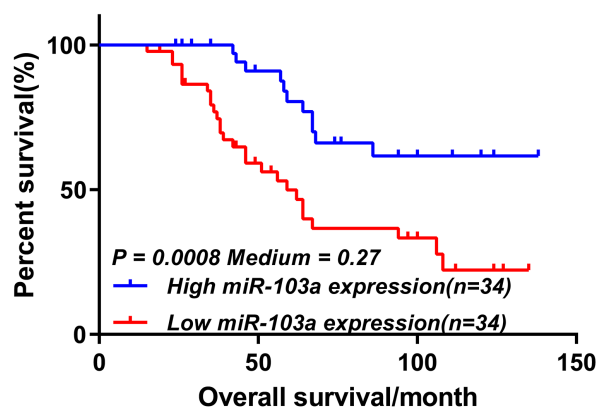

E

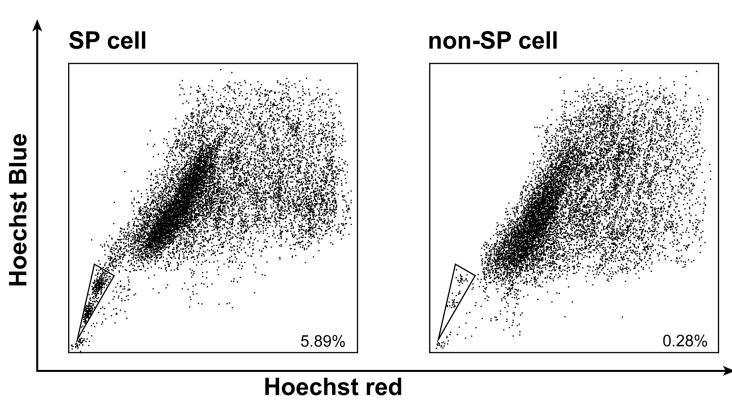

C
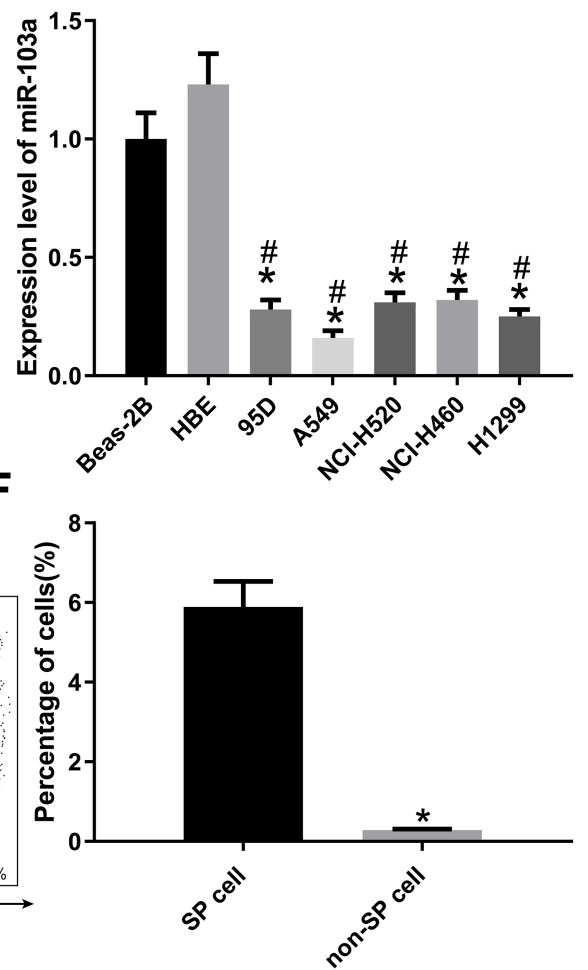

\section{Figure 1}

miR-103a is reduced in NSCLC tissues and cells and predicts good prognosis. A, the miR-103a expression in NSCLC and adjacent normal tissues determined by RT-qPCR ( ${ }^{\star} \mathrm{p}<0.05$ according to the two-way ANOVA); B, Kaplan-Meier analysis of the survival rate of NSCLC patients with high (blue) or low (red) miR103a expression; C, the miR-103a expression in Beas-2B, HBE, 95D, A549, NCl-H520, NCl-H460 and H1299 cells determined by RT-qPCR ( ${ }^{*} p<0.05$ according to the two-way ANOVA); D, observation of SP cells and 
non-SP cells morphology under a contrast microscope; E, SP and non-SP cells sorted by flow cytometry; F, the statistical analysis of panel $\mathrm{E}\left({ }^{*} \mathrm{p}<0.05\right.$ according to the unpaired $\mathrm{t}$ test). Each reaction was run in triplicate.

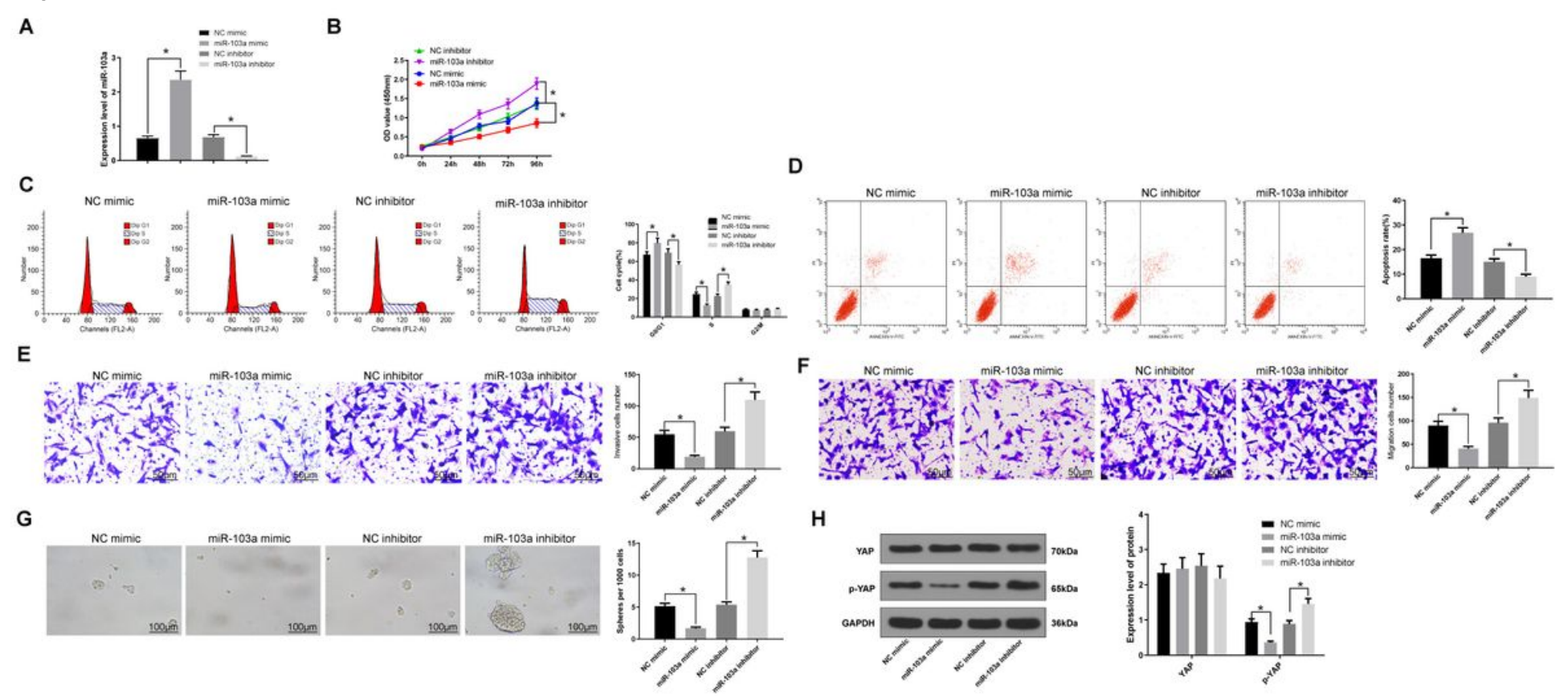

Figure 2

Ectopic expression of miR-103a attenuates the stemness of NSCLC cells. CSCs were delivered with miR103a mimic or inhibitor with NC mimic or inhibitor as controls. A, the miR-103a expression in cells after transfection determined by RT-qPCR ( ${ }^{*} p<0.05$ according to the one-way ANOVA); B, OD value of cells at the 0th, 24th, 48th, 72nd, and 96th $h$ measured by MTS assay ( ${ }^{*} p<0.05$ according to the two-way ANOVA); C, cell cycle distribution measured by flow cytometry ( ${ }^{*} \mathrm{p}<0.05$ according to the one-way ANOVA); $D$, cell apoptosis evaluated by flow cytometry ( ${ }^{\star} p<0.05$ according to the one-way ANOVA); $E$, cell invasion tested by Matrigel invasion assay ( ${ }^{*} \mathrm{p}<0.05$ according to the one-way ANOVA); F, cell migration examined by migration assay ( ${ }^{*} \mathrm{p}<0.05$ according to the one-way ANOVA); $\mathrm{G}$, sphere-formation activities of cells detected by sphere-formation assay ( ${ }^{*} p<0.05$ according to the one-way ANOVA); $H$, the expression of YAP and the extent of YAP phosphorylation measured by western blot ( ${ }^{*} \mathrm{p}<0.05$ according to the two-way ANOVA); the experiment was repeated 3 times independently. 
A

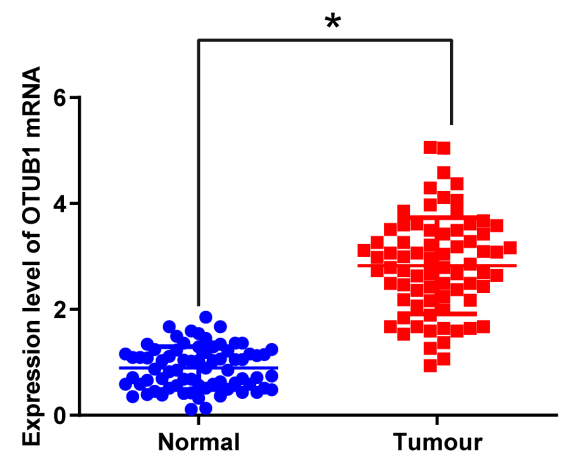

D

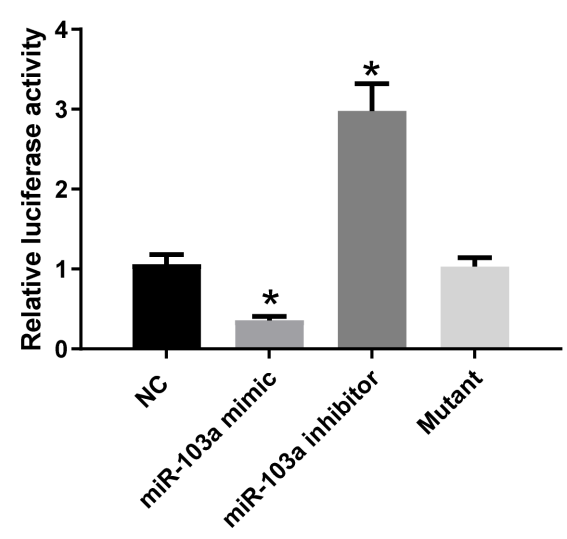

B

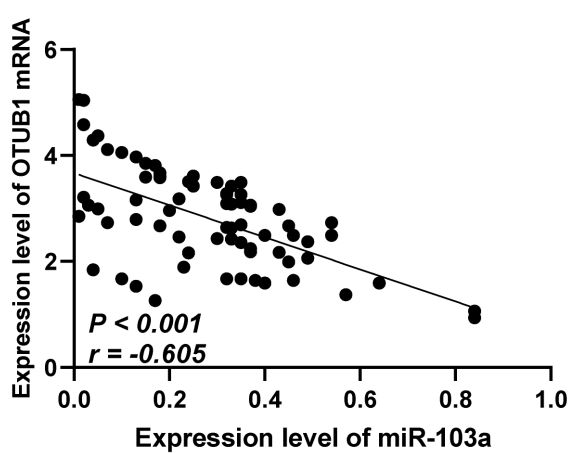

E

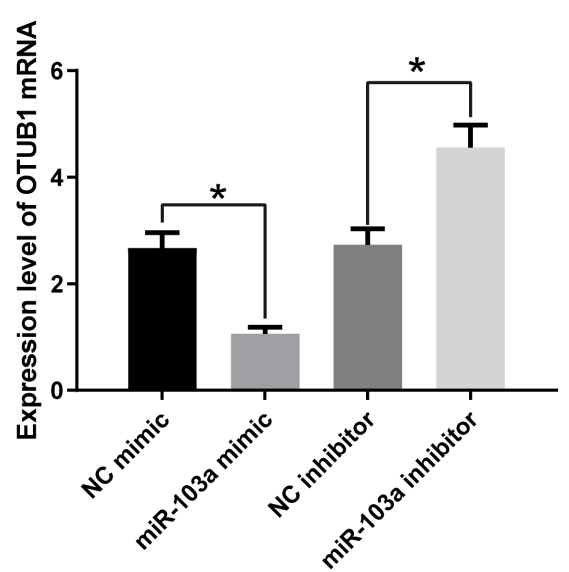

C

Alignment

Target: 5' ggcUGGCUCCAGCCCGCUGCUGCc 3'

|: || || | |: ||||||

miRNA: 3' aguAUCG GGACAUGUUACGACGa 5

$\mathbf{F}$

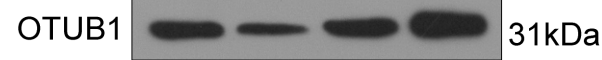

GAPDH $36 \mathrm{kDa}$

G
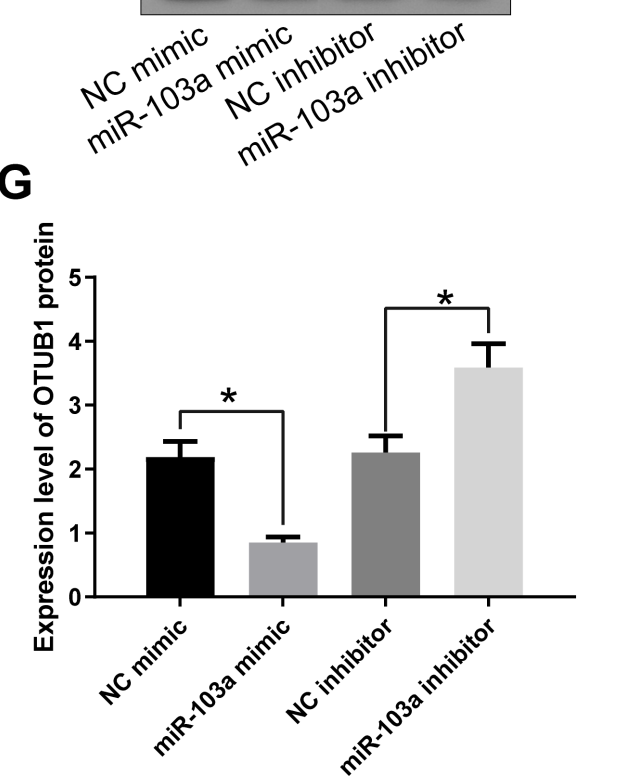

Figure 3

miR-103a targets and negatively controls OTUB1 in NSCLC cells. A, the mRNA expression of OTUB1 in NSCLC and adjacent normal tissues determined by RT-qPCR ( ${ }^{*} p<0.05$ according to the two-way ANOVA); $B$, the correlation analysis of miR-103a and OTUB1 mRNA in tissue samples of NSCLC; $C$, the sequences of miR-103a binding sites in OTUB1 3'UTR; D, the analysis of relative luciferase activities of PGLO-OTUB1 in cells treated with miR-103a mimic, inhibitor or mutant ( ${ }^{*} \mathrm{p}<0.05$ according to the one-way ANOVA); $\mathrm{E}$, mRNA expression of OTUB1 determined by RT-qPCR ( ${ }^{*} p<0.05$ according to the one-way ANOVA); $F$, protein expression of OTUB1 determined by western blot assays; $G$, the statistical analysis of panel $F$ ( ${ }^{*} p$ $<0.05$ according to the one-way ANOVA); the experiment was repeated 3 times independently. 

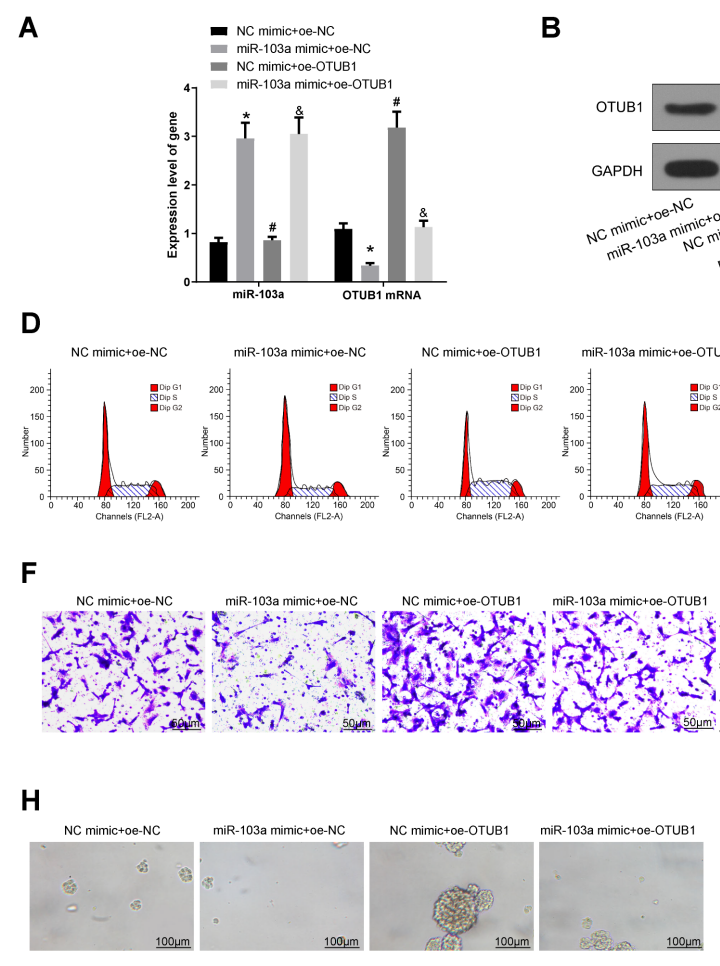

B

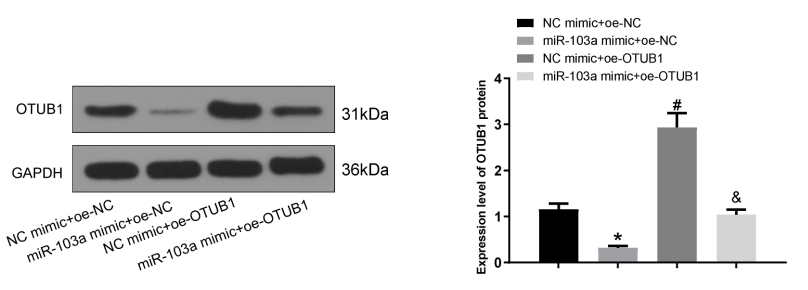

E
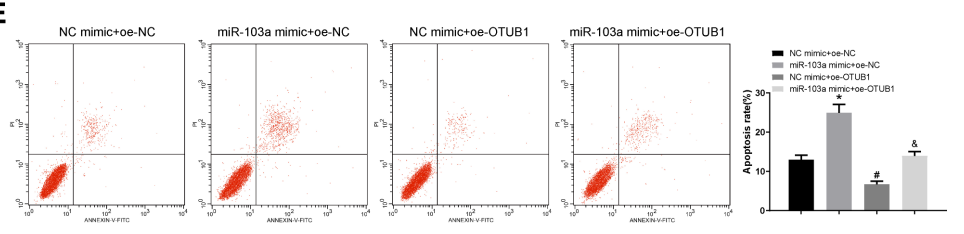

G

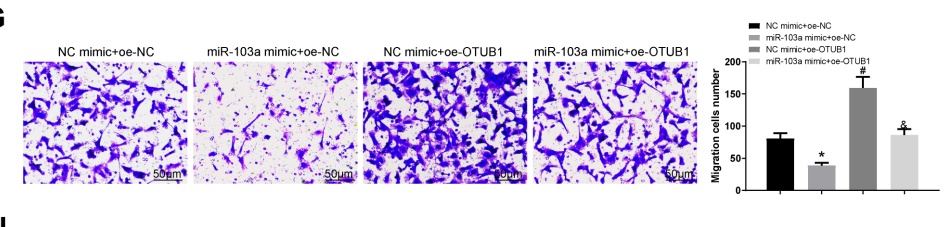

C $\quad \rightarrow$ NC mimic+oe-NC

2.57 miR-103a mimic+oe-OTUB1

$\bar{E}^{2.0}$

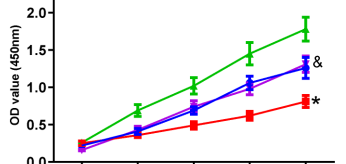

oh $\quad 24 \mathrm{~h} \quad 48 \mathrm{~h} \quad 72 \mathrm{~h} \quad 96 \mathrm{~h}$

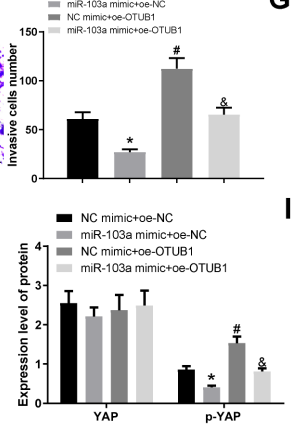

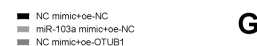
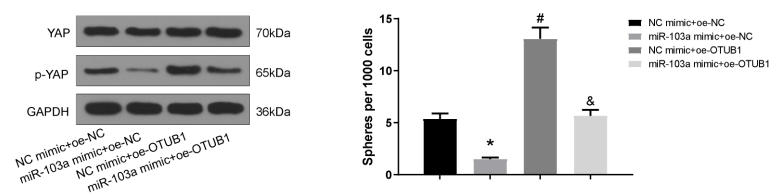

Figure 4

Overexpression of OTUB1 impairs miR-103a-ekoved inhibition of NSCLC cell stemness. Cells were treated miR-103a mimic and/or oe-OTUB1. A, miR-103a expression and mRNA expression of OTUB1 determined by RT-qPCR ( ${ }^{*} p<0.05$ according to the two-way ANOVA); B, the protein expression of OTUB1 determined by western blot assays ( ${ }^{*} p<0.05$ according to the one-way ANOVA); C, OD value of cells at the 0th, 24th, 48th, 72nd, and 96th $\mathrm{h}$ measured by MTS assay ( ${ }^{\star} \mathrm{p}<0.05$ according to the two-way ANOVA); D, cell cycle distribution measured by flow cytometry ( ${ }^{*} \mathrm{p}<0.05$ according to the two-way ANOVA); E, cell apoptosis evaluated by of flow cytometry ( ${ }^{*} \mathrm{p}<0.05$ according to the one-way ANOVA); F, cell invasion tested by Matrigel invasion assay ( ${ }^{*} \mathrm{p}<0.05$ according to the one-way ANOVA); G, cell migration examined by migration assay ( ${ }^{*} p<0.05$ according to the one-way ANOVA); $\mathrm{H}$, sphere-formation activities of cells detected by sphere-formation assay ( ${ }^{*} p<0.05$ according to the one-way ANOVA); l, the expression of YAP and the extent of YAP phosphorylation measured by western blot $\left({ }^{\star} \mathrm{p}<0.05\right.$ according to the two-way ANOVA). The experiment was repeated 3 times independently. 

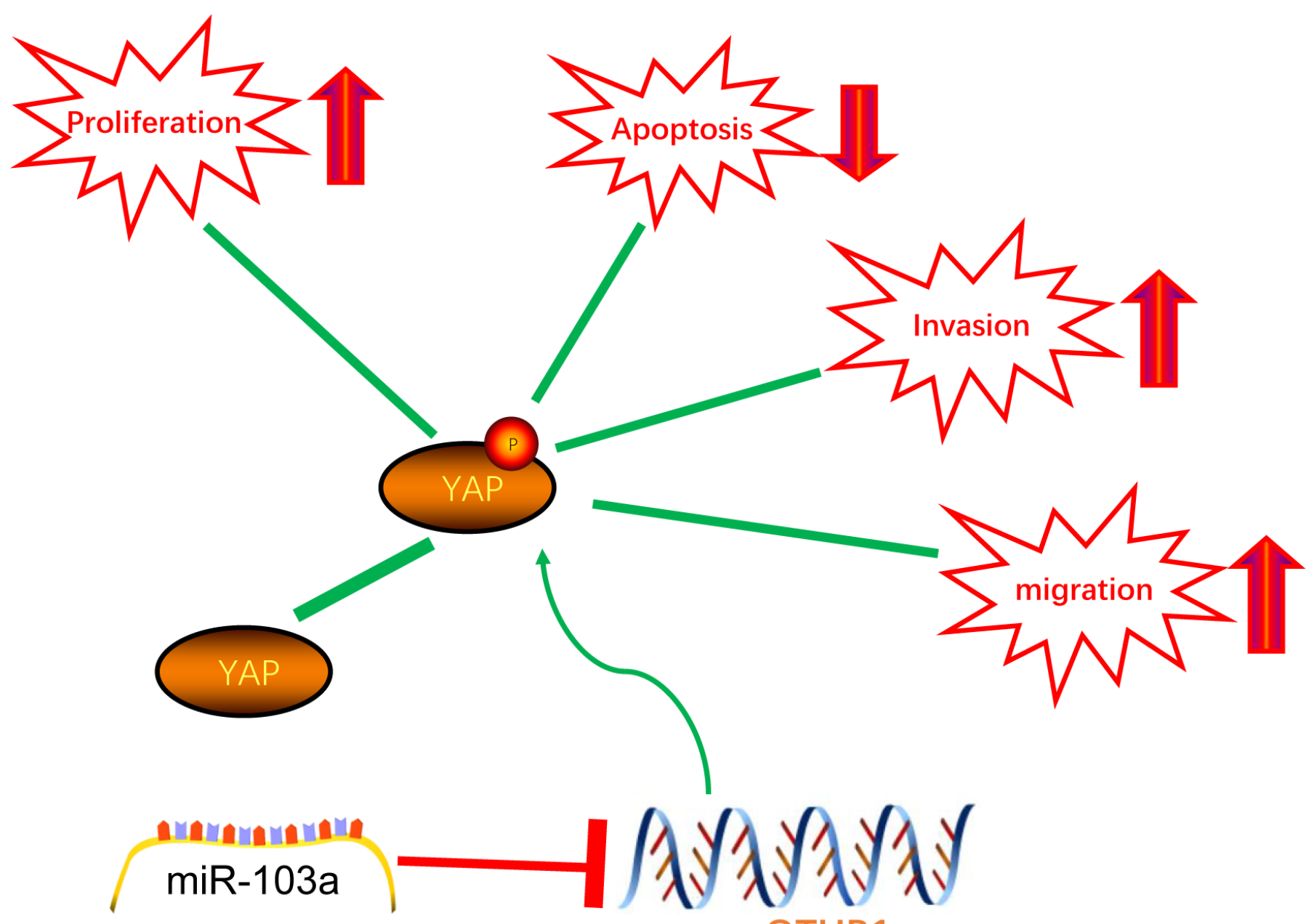

OTUB1

Figure 5

The schematic cartoon of the mechanism of miR-103a as a tumor suppressor by modulating the OTUB1/Hippo signaling pathway in CSCs in NSCLC. After YAP phosphorylation, Hippo signaling pathway is blunted to promote proliferation, migration, invasion and sphere formation of CSCs and to inhibit their apoptosis in NSCLC. Overexpression of miR-103a inhibits the expression of OTUB1 and induces the Hippo signaling pathway, thereby inhibiting the stemness of CSCs in NSCLC. 